\title{
Integral temperature hybrid laser sensor
}

\author{
C. A. Galindez , L. Rodriguez-Cobo, F. Anabitarte and J M. Lopez-Higuera \\ Photonics Engineering Group, Universidad de Cantabria \\ Av. Castros s/n, 39005-Santander, Spain.
}

\begin{abstract}
An integral temperature sensor based on Brillouin laser ring that is feed by a Fourier Domain mode-locking (FDML) laser is here proposed. The source FDML laser ring emits at $1532 \mathrm{~nm}$ within a range of $5 \mathrm{~nm}$. The working wavelength is given by tuning the offset voltage applied to a Fabry-Perot tunable filter (FFP-TF). In the present work, the FDML laser linewidth is set at $0.136 \mathrm{~nm}$. This linewidth allows a more efficient Brillouin response in the optical fiber without increasing the Brillouin threshold. The FDML laser linewidth is controlled by setting the amplitude modulation of the drive signal offset applied on the FFP-TF.
\end{abstract}

Keywords: Brillouin frequency shift, Fabry-Perot ring laser, tunable wavelength laser

\section{INTRODUCTION}

Laser schemes have been used as sensing devices because they are able to offer better signal to noise ratios and good sensitivities ${ }^{1}$. Fiber ring lasers based on Brillouin scattering are a well known laser configuration that have been intensively studied due to their narrow linewidth, gain quality of the nonlinear effect and easy experimental configuration, among other advantages. This kind of laser also offers the possibility to use fiber spools as active mediums within the cavity laser and then integral temperature sensors can be developed ${ }^{2}$. On the other hand, there are fiber ring lasers that are based on a Fabry-Perot tunable filter (FFP-TF), which can be easily tuned using bias voltage tuning ${ }^{3,4}$. The Fourier domain mode-locking (FDML) laser has become an attractive and powerful light source method in the field of optical sensor systems, since FDML lasers allow high speed and narrowband optical frequency sweeps.

In FDML, a managed delay line is incorporated into the laser cavity and a narrowband optical filter is tuned periodically at the cavity roundtrip time. This produces a quasi-stationary mode of operation. Light from one frequency sweep propagates through the cavity and returns to the filter at the exact time when the transmission window of the optical bandpass filter is tuned to the same optical frequency. Consequently, light from the previous round-trip is coupled back to the gain medium and lasing does not have to emerge from spontaneous emission. The narrowband optical bandpass filter dissipates almost no energy because the back-coupled light contains only frequencies that are matched to the transmission window filter at each moment. To obtain the same result in frequency domain, this involves destructive interference of all longitudinal modes that are not transmitted through the narrowband filter at a given time. Thus, the phases of the longitudinal modes must be locked. The laser output is not a train of short pulses; instead, it is a train of frequency sweeps and very long pulses. The tunable narrowband filtering is equivalent to an infinite number of narrowband amplitude modulators that are slightly out of phase.

In this paper, the light source for a Brillouin fiber ring laser is obtained from a FDML laser, and the Brillouin scattering signal is used to determine integral temperature variations of an optical fiber. In this case, the linewidth of the source is set around $0.136 \mathrm{~nm}$ at $1532 \mathrm{~nm}$.

\section{OPERATION PRINCIPLE AND EXPERIMENTAL SETUP}

The hybrid laser sensor configuration is shown in Fig. 1. It consists of two main sections; the right part of the setup (in blue) is the FDML laser source, and the left part of the figure (in black) is the Brillouin laser ring.

*galindezca@unican.es; phone/fax +34 942 200-877 

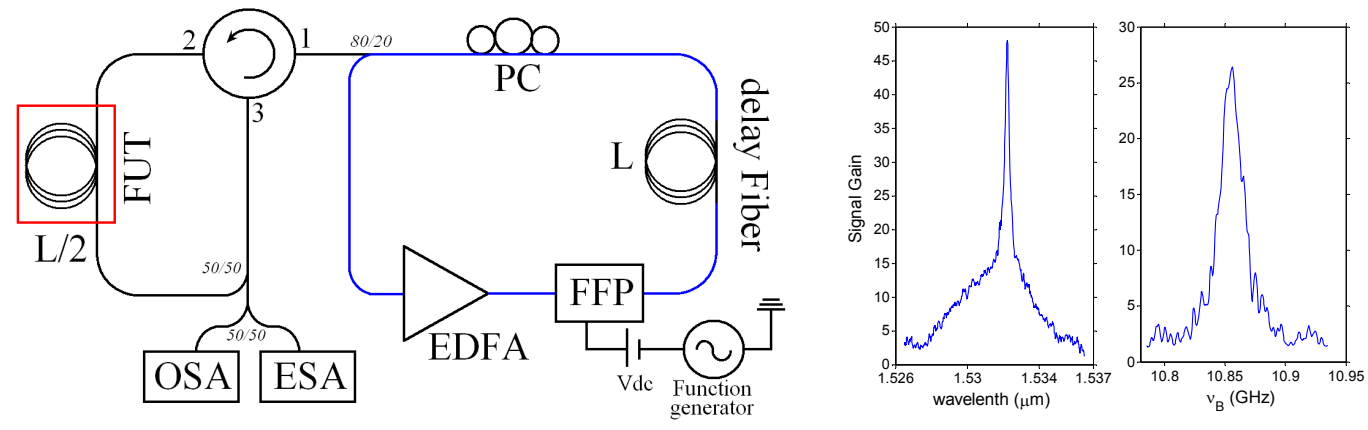

Figure 1. Sensor configuration (left figure). Optical signal and Brillouin frequency shift from the Brillouin laser ring.

The FDML laser is based on a fiber-ring geometry with an Erbium Doped Fiber Amplifier (EDFA) in the C-band as the gain medium, a fiber Fabry-Perot filter (FFP-TF, micron Optics, Inc) as the tunable narrowband optical bandpass filter, a polarization controller (PC) and the delay fiber as the delay medium to balance the sensor system. In order to have FDML operation, light with a certain wavelength must be transmitted by the filter again after one round trip. The time duration while the filter transmits certain wavelength determines the FDML wavelength width. The narrowband optical bandpass filter is driven periodically with a period matched to the optical roundtrip time of the laser cavity. In this work, the FDML is implemented with a physical cavity length (L) of $25 \mathrm{~km}$ of a commercial single mode optical fiber. This cavity length corresponds to optical roundtrip frequency of $11.05 \mathrm{kHz}$ with a peak-to-peak voltage of $100 \mathrm{mV}$ to obtain a $0.136 \mathrm{~nm}$ of linewidth. The FFP-TF is periodically tuned at half of the cavity roundtrip frequency, since the signal used has sinusoidal shape, where the rise and falling edges have to be considered.

The Brillouin fiber laser sensor is also based on fiber-ring geometry with a spool of fiber as the active medium and the transducer. The optical signal coming from the third port of the circulator 1 (C1) is split equally; one of the output signals is analyzed by mean of an optical spectrum analyzer (OSA) to measure the wavelength directly, while the other output is converted to an electrical signal by using a photodiode with $20 \mathrm{GHz}$ of bandwidth, then the signal is analyzed by using an electrical spectrum analyzer (ESA). A sample of the Brillouin laser ring signal measured with the OSA and the ESA is depicted in the right part of Fig. 1.

\section{EXPERIMENTAL RESULTS}

The optical signal generated in the FDML laser is used as light source for the ring cavity, which is depicted in the left side of the setup configuration figure (Fig. 1). The optical signal that comes from the FDML laser starts the spontaneous Brillouin scattering. Fig. 2 shows the behavior of the Brillouin ring laser for frequency drive values around the half roundtrip frequency. The upper figure exposes the optical gain of the Brillouin scattering and the lower figure shows the Brillouin frequency shift. From the Fig. 2, it can be noted that the Brillouin laser works for the same frequencies of resonance than the FDML laser does.

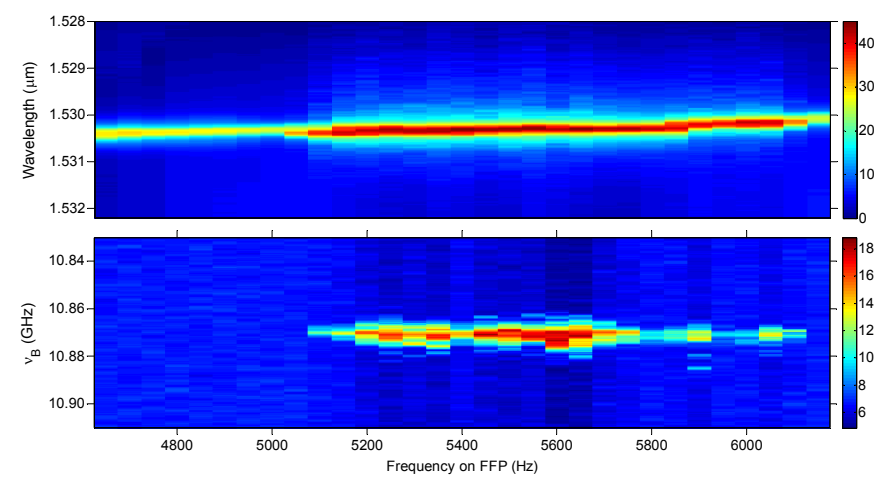

Figure 2. Wavelength (upper figure) and Brillouin frequency (lower figure) response of the Brillouin ring cavity with the half drive frequency. 


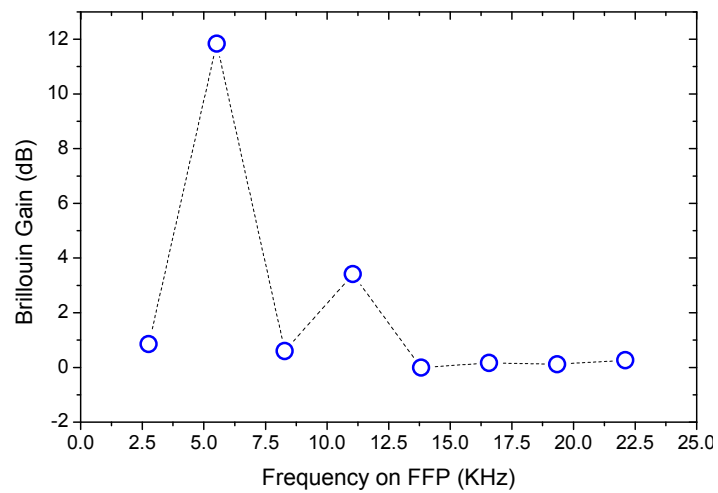

Figure 3. Brillouin frequency shift gain response of the Brillouin ring cavity with multiples of the drive frequency.

For demonstrating that the Brillouin ring laser only emits at certain drive frequencies, 8 values of drive frequency were programmed in the function generator. Fig. 3 shows the results, it clearly exposes that the Brillouin gain is only reached for $11.05 \mathrm{kHz}$ and $11.05 / 2 \mathrm{kHz}$.

Additionally to the drive frequency determination, the FDML laser has to be characterized respect to the drive voltage, for finding the optimum Vbias that maximize the optical power transmitted to the Brillouin ring laser. The characterization is depicted in Fig. 4; it shows the linear dependence with the drive voltage variation. From this figure the maximum Brillouin gain value can be obtained. Thus, the maximized FDML laser gives a Brillouin ring laser working at $1532 \mathrm{~nm}$ and $10.85 \mathrm{GHz}$ of Brillouin frequency shift.
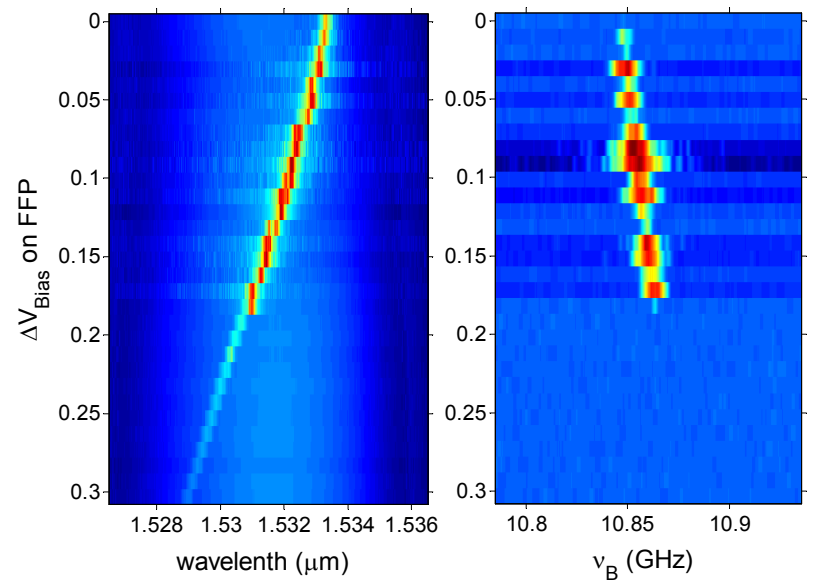

Figure 4 Wavelength (left figure) and Brillouin frequency (right figure) response of the Brillouin ring cavity with the bias voltage at the drive frequency. $(0.09279 \mathrm{GHz} / \mathrm{V}$ and $-13.581 \mathrm{~nm} / \mathrm{V})$.

Now the laser sensor is fully characterized and perfectly working, the system can be used for measuring the temperature all over the fiber under test (See Fig. 1). Since it is well known that the Brillouin scattering depends on the fiber temperature, the Brillouin frequency shift from the Brillouin ring cavity variations can be measured to determine the fiber temperature. The response of the Brillouin frequency with the temperature is shown in Fig. 5. Furthermore, by using this sensor configuration the temperature can be measured in real time by means of the ESA ${ }^{2}$. This sensors configuration can also be implemented with a semiconductor optical amplifier (SOA) in order to obtain a flatter and wider wavelength range of operation, or with an EDFA working in L-band. 


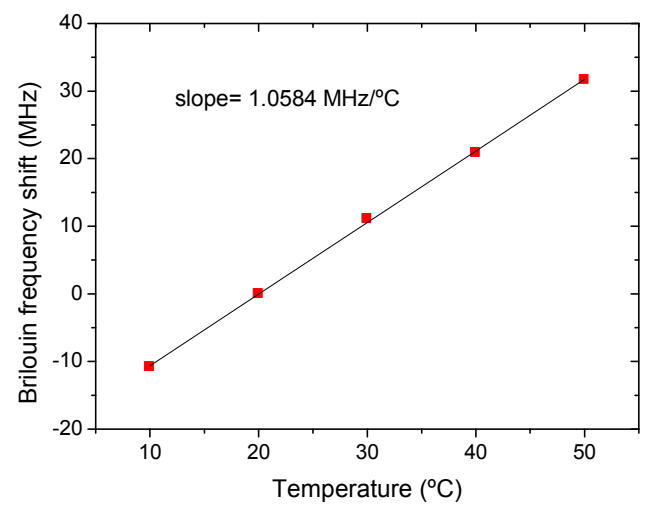

Figure 5. Brillouin frequency shift of the Brillouin ring cavity with temperature variations.

\section{CONCLUSIONS}

In this paper an integral temperature sensor based on Brillouin laser ring and fed by a FDML laser is proposed and demonstrated. The source FDML laser ring was optimized for emitting at $1532 \mathrm{~nm}$ within a range of $5 \mathrm{~nm}$ and a laser linewidth of $0.136 \mathrm{~nm}$. The FDML laser linewidth is controlled by setting the amplitude modulation of the offset driven signal applied on the FFP-TF. This method avoids the usage of electro-optic modulators, tunable lasers and allows the use of strong signals to determine the frequency variations. This active sensor system shows a good accuracy and can be used in rough environments for real time measurement. To extend the integral measurement to several sensing areas, different optical fibers (one per area) can be serially concatenated.

\section{ACKNOWLEDGMENTS}

This work has been supported by the Spanish TEC2010-20224-C02-02 project.

\section{REFERENCES}

[1] Lopez-Higuera, J. M., Handbook of optical fibre sensing technology: John Wiley \& Sons Inc, 2002.

[2] C. A. Galindez, F. J. Madruga, A. Ullan, M. Lopez-Amo, and J. M. Lopez-Higuera, "Temperature sensing in multiple zones based on Brillouin fiber ring laser," Journal of Physics: Conference Series 178, 1-5 (2009).

[3] Y. Yeh, D. Park, and S. H. Park, "High-speed measurement of free spectral range voltage of tunable filters," Opt. Lett. 34, 52-54 (2009).

[4] E. J. Lee and Y. P. Kim, "Wavelength-tunable and pulse-width variable Fourier domain mode-locking lasers," Opt. Lett. 36, 4752-4754. 